\title{
Lumbar cerebrospinal fluid-to-brain extracellular fluid surrogacy is context-specific: insights from LeiCNS-PK3.0 simulations
}

\author{
Mohammed A. A. Saleh ${ }^{1}$ (1) $\cdot$ Chi Fong Loo ${ }^{1} \cdot$ Jeroen Elassaiss-Schaap ${ }^{1,2}$ (1) $\cdot$ Elizabeth C. M. De Lange ${ }^{1}$ (D)
}

Received: 12 January 2021 / Accepted: 1 June 2021 / Published online: 17 June 2021

(C) The Author(s) 2021

\begin{abstract}
Predicting brain pharmacokinetics is critical for central nervous system (CNS) drug development yet difficult due to ethical restrictions of human brain sampling. CNS pharmacokinetic (PK) profiles are often altered in CNS diseases due to diseasespecific pathophysiology. We previously published a comprehensive CNS physiologically-based PK (PBPK) model that predicted the PK profiles of small drugs at brain and cerebrospinal fluid compartments. Here, we improved this model with brain non-specific binding and $\mathrm{pH}$ effect on drug ionization and passive transport. We refer to this improved model as Leiden CNS PBPK predictor V3.0 (LeiCNS-PK3.0). LeiCNS-PK3.0 predicted the unbound drug concentrations of brain ECF and CSF compartments in rats and humans with less than two-fold error. We then applied LeiCNS-PK3.0 to study the effect of altered cerebrospinal fluid (CSF) dynamics, CSF volume and flow, on brain extracellular fluid (ECF) pharmacokinetics. The effect of altered CSF dynamics was simulated using LeiCNS-PK3.0 for six drugs and the resulting drug exposure at brain ECF and lumbar CSF were compared. Simulation results showed that altered CSF dynamics changed the CSF PK profiles, but not the brain ECF profiles, irrespective of the drug's physicochemical properties. Our analysis supports the notion that lumbar CSF drug concentration is not an accurate surrogate of brain ECF, particularly in CNS diseases. Systems approaches account for multiple levels of CNS complexity and are better suited to predict brain PK.
\end{abstract}

Keywords Physiologically-based pharmacokinetic models $\cdot$ CNS $\cdot$ Drug development $\cdot$ Brain

\section{Introduction}

Central nervous system (CNS) pharmacokinetic (PK) profiling, though challenging, remains critical for drug development. Two PK profiles can be distinguished in the CNS: brain and cerebrospinal fluid (CSF) PK profiles. In CNS drug development, compounds are selected that optimize brain PK profile, since brain cells and extracellular fluid (ECF) represent the major site of drug (side-) effects. Suboptimal drug exposure in brain has resulted in clinical trial failure and has ultimately contributed to the high attrition rate of the CNS drugs in development [1]. CSF represents a relatively accessible matrix to sample the CNS, mainly via lumbar puncturing. While lumbar CSF

Elizabeth C. M. De Lange

ecmdelange@lacdr.leidenuniv.nl

1 Division of Systems Biomedicine and Pharmacology, Leiden Academic Center for Drug Research, Leiden University, Leiden, The Netherlands

2 PD-Value B.V, Houten, The Netherlands drug concentrations predict brain concentrations better than that of plasma [2], its accuracy as a surrogate of brain PK has been argued [3], particularly for low passive permeability and actively transported drugs [4].

The major challenge in designing drugs with adequate brain PK, is the poor understanding of the role of CNS (patho)physiology in determining brain PK [5]. Up to this challenge, a mechanistic, systems-based understanding of key physiological and pathological processes in healthy and diseased CNS is instrumental in predicting brain (patho-) pharmacokinetics.

Our group previously published a comprehensive CNS physiologically-based (PBPK) model that predicts the unbound concentration-time profiles of small drugs within the CNS [6, 7]. This model, hereafter referred to as Leiden CNS PBPK predictor 1.0 (LeiCNS-PK1.0), was developed using knowledge-based, bottom-up modeling [6, 7], without using in vivo-measured PK profiles for model building. The mechanistic structure of LeiCNS-PK1.0 allows interspecies and interpopulation translation and provides a framework to study the effect of altering a single or 
multiple physiological aspects on CNS PK. Thus, LeiCNSPK1.0 can be used to predict mechanistically the effect of disease-altered CNS physiology on unbound drug exposure in brain [7]. While LeiCNS-PK1.0 could adequately predict the CNS PK profiles of rats and healthy humans [6, 7], several components of CNS physiology, including brain tissue non-specific binding and $\mathrm{pH}$ impact on passive transport, were represented in a rudimentary manner. This limited the translatability of LeiCNS-PK1.0 predictions between species and from healthy to diseased populations. First, the calculated $\mathrm{pH}$ factors did not reflect the neutral drug fraction of a given compartment, as neutral drug fraction in each compartment was normalized to that of the plasma compartment. In addition, it was assumed that the charged drug molecules do not undergo transcellular or paracellular passive transport across the blood-brain (BBB) and blood-cerebrospinal fluid (BCSFB) barriers, which is not physiologically plausible as charged drugs can be transported via the passive paracellular route [8]. Accounting for the impact of $\mathrm{pH}$ on drug ionization has been shown to clearly improve the prediction of CNS PK profiles of drugs with weak acidic and/or basic groups [9]. Drug non-specific binding, on the other hand, lacked a mechanistic description and physiological plausibility as it was assumed in LeiCNS-PK1.0 to occur instantaneously within the ECF and was calculated using the unbound drug fraction in brain and plasma, brain tissue composition, and lipophilicity of the drug. Brain unbound drug fraction $\left(\mathrm{f}_{\mathrm{u}, \mathrm{b}}\right)$ as measured in vitro, varies between measurement techniques, requires brain tissue, and might not be available at early stages of drug development. Brain non-specific binding has been demonstrated to be one of the major determinants of brain pharmacokinetics [10], particularly for lipophilic drugs [11-14]. Hence, LeiCNS-PK1.0 required improvement.

In this paper, we first improve LeiCNS-PK1.0 by readdressing the effect of $\mathrm{pH}$ on drug ionization, LeiCNSPK1.0 assumptions related to passive transport of charged molecules at BBB and BCSFB barriers, and the time-dependent brain tissue non-specific binding. We refer to this improved model as Leiden CNS PBPK predictor 3.0 or LeiCNS-PK3.0. Next, we use LeiCNS-PK3.0 model to explore the effect of altered CSF dynamics on CSF and brain ECF PK profiles as well as on predictability of brain ECF drug concentration by that of lumbar CSF. Changes in CSF dynamics, CSF volume and flow, are common in CNS diseases (Table 1) and often alter CSF PK; their effect on the brain ECF PK profiles remains unexplored [15].

\section{Methods}

\section{CNS and plasma in vivo-measured drug concentrations}

Drugs used to validate the model predictions included acetaminophen, atenolol, methotrexate, morphine, phenytoin, raclopride, risperidone, paliperidone, remoxipride, quinidine, oxycodone, and indomethacin. These drugs were selected to cover the physicochemical space of small drug molecules with molecular weights between 100 and $500 \mathrm{~g} / \mathrm{mol}$, different ionization rate constants and charge class at physiological $\mathrm{pH}$, different lipophilicity, and different affinity to active transporters at the $\mathrm{BBB}$ and BCSFB.

Plasma PK data, for the development of the empirical plasma models, and CNS PK data, for the evaluation of LeiCNS-PK3.0 predictions, were available for both rats and humans from the literature. Supplementary table 1 summarizes the sampling location and data references.

For validating the rat version of LeiCNS-PK3.0, only inhouse data were used, where individual unbound PK profiles were simultaneously measured in the same animal under controlled conditions in plasma and in multiple CNS locations: brain ECF, lateral ventricles (LV), and cisterna magna $(\mathrm{CM})$ using microdialysis, in addition to total brain concentrations, which were measured with the brain homogenate method. Clinical brain PK profiles measured with microdialysis are quite rare due to ethical restrictions. In humans, individual unbound PK profiles of brain ECF and lumbar CSF were available from patients with conditions that do not affect CNS physiology or from healthy, uninjured sites. Acetaminophen and indomethacin concentrations were measured in patients with nerve root compression. Oxycodone were available from patients undergoing elective gynecological surgery. Morphine concentrations were collected using microdialysis from uninjured brain tissue sites from traumatic brain injury patients.

Total drug concentrations were corrected using respective fraction of unbound drug where needed. CSF drug concentrations were assumed unbound due to the low protein content of the CSF, i.e. $\mathrm{f}_{\mathrm{u}, \mathrm{CSF}}=1$, except for indomethacin with an $\mathrm{f}_{\mathrm{u}, \mathrm{CSF}}$ of 0.47 [16].

\section{Drug-specific parameters}

Drug specific parameters: lipophilicity $\left(\log \mathrm{P}_{\mathrm{o} / \mathrm{w}}\right)$, acid/base ionization constants $\left(\mathrm{pK}_{\mathrm{a}} / \mathrm{pK}_{\mathrm{b}}\right)$, and molecular weight, were collected from Drugbank [17] and are listed in Table 2. Calculated $\log \mathrm{P}_{\mathrm{o} / \mathrm{w}}$ values by ALOGPS method [18] were used, unless experimental $\log \mathrm{P}_{\mathrm{o} / \mathrm{w}}$ values were 
Table 1 Cerebrospinal fluid dynamics in different CNS disease conditionsa

\begin{tabular}{lllll}
\hline & Aging $^{\mathrm{a}}$ & Alzheimer's disease & Hydrocephalus & Traumatic brain injury $^{\mathrm{a}}$ \\
\hline CSF volume & $400 \%$ & $150 \%^{\mathrm{b}}$ & $150 \%^{\mathrm{b}}$ & $115 \%$ \\
& {$[51]$} & {$[52]$} & {$[53]$} & {$[54]$} \\
CSF production & $66 \%$ & $46 \%^{\mathrm{a}}$ & $60 \%^{\mathrm{b}}$ \\
& {$[55]$} & {$[56]$} & {$[57]$} \\
CSF flow & $150 \%$ & Normal CSF flow & $370 \%$ and reverse flow direction $^{\mathrm{a}}$ & \\
& {$[58]$} & {$[59]$} & {$[32]$} \\
CSF clearance & Reduced CSF absorption & & \\
& {$[60]$} & $65 \%^{\mathrm{b}}$ & {$[61]$} & {$[62]$} \\
\hline
\end{tabular}

${ }^{\mathrm{a}}$ Compared to adults $(<60$ years)

${ }^{\mathrm{b}}$ Compared to elderly $(60+$ years $)$

${ }^{\mathrm{c}} \mathrm{A}$ study in rats

available, while calculated $\mathrm{pK}_{\mathrm{a}} / \mathrm{pK}_{\mathrm{b}}$ values by the MARVIN method provided by CHEMAXON [19] were used.

\section{Leiden CNS PBPK predictor V3.0 (LeiCNS-PK3.0)}

\section{Model development}

LeiCNS-PK3.0 (Fig. 1 and Supplementary Fig. 1) consists of an empirical plasma model, which predicts plasma PK, and a nine-compartment CNS model. The empirical plasma model serves as an input that drives the PK of the CNS model, with both models linked by the cerebral blood flow. Development of the empirical plasma model and detailed description of the CNS model structure, physiological processes, and transport modes are described below. The physiological parameters of rats and humans are presented in Supplementary table 2. When multiple values were found in the literature, the mean value was used.

LeiCNS-PK3.0 is an improvement of the published LeiCNS-PK1.0 [6, 7] on aspects related to brain nonspecific binding, $\mathrm{pH}$ effect on drug ionization, and assumptions related to transcellular and paracellular passive diffusion of the charged drug molecules. A comparison of the improved aspects in LeiCNS-PK3.0 compared to LeiCNS-PK1.0 is presented in Table 3.

\section{CNS compartments}

In LeiCNS-PK3.0, different CNS compartments are accounted for: brain microvessels, brain extracellular fluid (ECF), brain intracellular fluid (ICF), lysosomes, cranial cerebrospinal fluid (CSF) compartments: lateral ventricles, third and fourth ventricles, and cisterna magna, in addition to the CSF in subarachnoid space (SAS), including lumbar CSF. A new compartment, brain cell membrane, has been added to LeiCNS-PK3.0, as the assumed non-specific binding site in brain.

\section{$\mathrm{pH}$ effect on drug ionization}

The $\mathrm{pH}$ factors (PHF) are defined as the neutral fraction of the drug concentration of a given compartment. PHF is determined using adapted Henderson-Hasselbalch equations utilizing compartment-specific $\mathrm{pH}\left(\mathrm{pH}_{\mathrm{comp}}\right)$ and the ionization constants of the strongest acidic group $\left(\mathrm{pk}_{\mathrm{a}}\right)$ and the strongest basic group $\left(\mathrm{pk}_{\mathrm{b}}\right)$ of the drug. In case of drugs missing one group (e.g. risperidone has only a basic group, but no acidic groups), the relevant neutral fraction of this missing group is set to 1 . PHF is calculated as per the equations below.

Neutral fraction of acidic group $\left(P H F_{\text {acidic }}\right)=\frac{1}{1+10^{p H_{c o m p}-p k_{a}}}$

Neutral fraction of basic group $\left(P H F_{\text {basic }}\right)=\frac{1}{1+10^{p k_{b}-p H_{\text {comp }}}}$

Neutral fraction of $\operatorname{drug}(P H F)=P H F_{\text {acidic }} \times P H F_{\text {basic }}$

\section{Brain tissue non-specific binding}

In LeiCNS-PK3.0, brain phospholipids, which constitute a major fraction of brain cell membranes, are assumed as the non-specific binding site in brain [20-22]. The volume of the brain cell membrane compartment is $5 \%$ of the total brain volume, which represents the volume fraction of phospholipids in the brains of rats [23] and humans [24]. $\mathrm{CL}_{\mathrm{wo}}$ and $\mathrm{CL}_{\mathrm{ow}}\left(\mathrm{mL} \min ^{-1}\right)$ describe the diffusion clearance of a given drug between brain ECF and ICF on one side and brain cell membrane on the other side. At steady state, the ratio of the drug concentration in the brain cell 


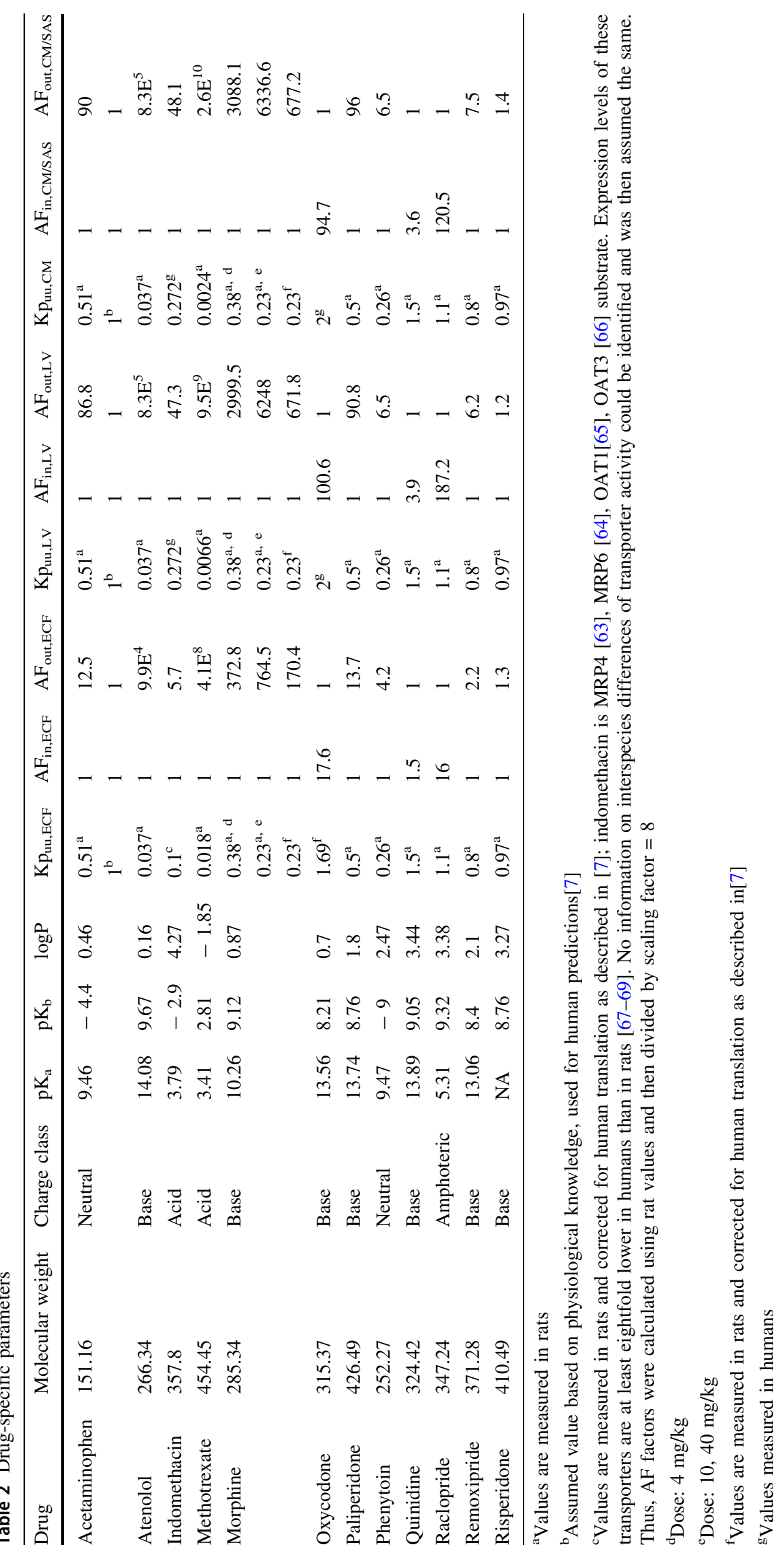




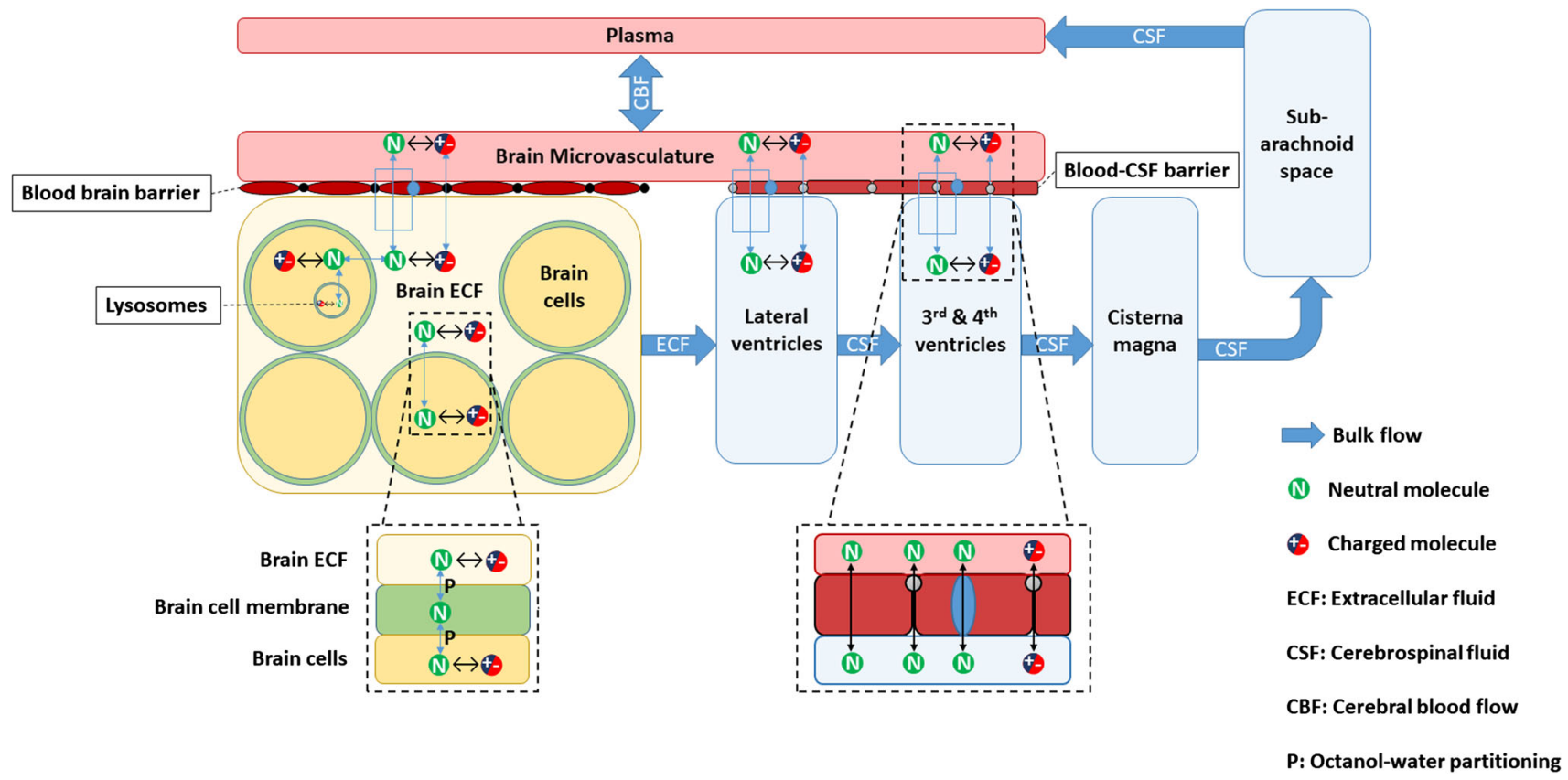

Fig. 1 LeiCNS-PK3.0 model structure. LeiCNS-PK3.0 is composed of a whole body empirical plasma model and a CNS PBPK model. Both models are connected via cerebral blood flow

Table 3 Comparison of the improved aspects in LeiCNS-PK3.0 versus LeiCN-PK1.0

\begin{tabular}{|c|c|c|}
\hline Aspect & LeiCNS-PK1.0 & LeiCNS-PK3.0 \\
\hline \multirow[t]{2}{*}{$\mathrm{pH}$ factor (PHF) } & $\begin{array}{l}\text { Defined as the ratio of the neutral fraction of a drug of a given } \\
\text { compartment to that of plasma }\end{array}$ & $\begin{array}{l}\text { Defined as the neutral fraction of a drug in a given } \\
\text { compartment }\end{array}$ \\
\hline & $\begin{array}{l}\text { Calculated using Henderson-Hasselbalch equations with } \mathrm{pH} \text { of the } \\
\text { compartment, } \mathrm{pH} \text { of plasma, and the drug-specific ionization constant }\end{array}$ & $\begin{array}{l}\text { Calculated using adapted Henderson-Hasselbalch } \\
\text { equations using compartment specific } \mathrm{pH} \text { and the } \\
\text { drug-specific ionization constant }\end{array}$ \\
\hline \multirow{4}{*}{$\begin{array}{l}\text { Brain tissue } \\
\text { non-specific } \\
\text { binding }\end{array}$} & Using binding factor & Mechanistic description \\
\hline & Instantaneous & $\begin{array}{l}\text { According to diffusion clearance between aqueous } \\
\text { and lipid phases }\end{array}$ \\
\hline & Binding occurs within the brain ECF to a hypothetical compartment & $\begin{array}{l}\text { Binding occurs to the phospholipids of the brain } \\
\text { cell membrane }\end{array}$ \\
\hline & $\begin{array}{l}\text { Relies on total brain-to-plasma concentration ratio }\left(\mathrm{K}_{\mathrm{p}}\right) \text {. } \mathrm{K}_{\mathrm{p}} \text { is calculated } \\
\text { using drug lipophilicity }(\log \mathrm{P}) \text {, unbound drug fraction in brain }\left(\mathrm{f}_{\mathrm{u}, \mathrm{b}}\right) \\
\text { and plasma }\left(\mathrm{f}_{\mathrm{u}, \mathrm{p}}\right) \text {, and brain and plasma tissue composition }\end{array}$ & $\begin{array}{l}\text { Relies on drug lipophilicity and the volume of } \\
\text { brain phospholipids }\end{array}$ \\
\hline $\begin{array}{l}\text { Passive } \\
\text { paracellular } \\
\text { transport }\end{array}$ & Paracellular route is restricted to neutral drug only & $\begin{array}{l}\text { Paracellular route is available for both neutral and } \\
\text { charged drug }\end{array}$ \\
\hline
\end{tabular}

membrane to the drug concentration in the brain ECF and ICF is equal to the octanol-water partition coefficient $\left(\mathrm{P}_{\text {oct- }}\right.$ water).

\section{Bulk fluid flow}

Bulk fluid flow refers to the drug clearance between CNS compartments due to fluid flow, irrespective of the concentration gradients. In LeiCNS-PK3.0, bulk flows include cerebral blood flow between the brain microvessels and the central compartment of the empirical plasma model, ECF bulk flow from brain ECF to LV, and the CSF flow from the cranial CSF to the absorption sites in SAS.

\section{Passive transport}

Passive transport in the CNS involves paracellular and transcellular transport. Transcellular transport refers to the permeability of the drug through phospholipid bilayer of the membranes of the BBB endothelial cells, BCSFB 
epithelial cells, brain parenchyma, and lysosomes. Paracellular transport describes the aqueous diffusion of the drug molecules between the cells of the BBB and BCSFB via the openings of the tight junctions. Further details on the equations required to calculate aqueous diffusion and transmembrane permeability are reported in the supplementary information and in [6].

In LeiCNS-PK3.0, neutral drug molecules are transported through both transcellular and paracellular routes, whereas charged drug molecules are transported via paracellular routes only. Anions, cations, and zwitterions are assumed to undergo paracellular diffusion at the same rate.

\section{Asymmetry factors}

In LeiCNS-PK3.0, physiological processes that are not explicitly addressed such as active transport across the BBB and BCSFB, and metabolism, are accounted for using asymmetry factors (AF). AF were calculated using the LeiCNS-PK3.0 equations at steady state and $\mathrm{Kp}_{\mathrm{uu}}$, the ratio of the unbound drug concentration in a given tissue to that of plasma. $\mathrm{Kp}_{\mathrm{uu}}$ values were available from the literature or calculated using influx and efflux clearances of a given compartment [25].

$K p_{\text {uи }}=\frac{C l_{\text {in }}}{C l_{\text {out }}}$

where $\mathrm{Kp}_{\mathrm{uu}}$ is the ratio of unbound concentration of a given tissue compartment to that of plasma at steady state, $\mathrm{Cl}_{\text {in }}$ is the total influx clearance into the tissue compartment, and $\mathrm{Cl}_{\text {out }}$ total efflux clearance out of the tissue compartment. Influx and efflux clearances can be estimated using available unbound drug concentration-time profiles. In humans, $\mathrm{Kp}_{\mathrm{uu}}$ values are not often available and can be calculated as described in the decision tree presented in [7]. If in vivomeasured $\mathrm{Kp}_{\mathrm{uu}}$ values are unavailable, $\mathrm{AF}$ can be derived from in vitro estimates such as efflux ratio and cell uptake values as we described previously [7, 26].

Equations for calculating $\mathrm{AF}$ are provided in the supplementary materials. Influx $\mathrm{AF}\left(\mathrm{AF}_{\mathrm{in}}\right)$ and efflux $\mathrm{AF}$ $\left(\mathrm{AF}_{\mathrm{ef}}\right)$ are calculated at $\mathrm{BBB}, \mathrm{BCSFB}_{\mathrm{LV}}$, and $\mathrm{BCSFB}_{\mathrm{TFV}}$, where three scenarios are possible depending on the value of $\mathrm{Kp}$ uu. $\mathrm{Kp}_{\mathrm{uu}}$ equal to 1 suggests an equilibrium of drug concentration across $\mathrm{BBB} / \mathrm{BCSFB}$, and thus $\mathrm{AF}_{\text {in }}$ and $\mathrm{AF}_{\text {ef }}$ are equal to $1 . \mathrm{Kp}_{\mathrm{uu}}$ smaller than 1 suggests active efflux at $\mathrm{BBB} / \mathrm{BCSFB}$; in this case $\mathrm{AF}_{\text {in }}$ is set to 1 , while $\mathrm{AF}_{\text {ef }}$ is calculated using the relevant equation and the associated $K p_{u u}$ value. $K p_{u u}$ larger than 1 suggests active influx at $\mathrm{BBB} / \mathrm{BCSFB}, \mathrm{AF}_{\text {ef }}$ is set to 1 , and then $\mathrm{AF}_{\text {in }}$ is calculated [7].

The calculated AF values are listed in Table 2. The AF factors of atenolol and methotrexate were exceptionally high, which can be attributed mainly to their very low $\mathrm{Kp}_{\mathrm{uu}}$ values. Atenolol $\left(\mathrm{Kp}_{\mathrm{uu}}=0.037\right)$ is a low passive permeability molecule and recent evidence show that atenolol might undergo active transport at the BBB [27]. Methotrexate $\left(\mathrm{Kp}_{\mathrm{uu}}=0.018,0.0066,0.0024\right.$ for ECF, LV, and CM, respectively) is a substrate of PGP [28], BCRP [29], and MRP4 [30], which are three main transporters at the BBB and BCSFB. At CNS physiological $\mathrm{pH}$, methotrexate acts as an anion, whose negative charge could reduce its passive permeability as a result of the interaction with negatively charged phospholipids of the cell membranes. The combined low passive permeability and presence of active transport contribute to the low $\mathrm{Kp}_{\mathrm{uu}}$ of both drugs.

\section{Empirical plasma PK models}

Rat plasma PK models were developed using non-linear mixed effects modeling, where one-, two-, three- compartment models were compared. Interindividual variability was tested using an exponential model for every PK parameter. Residual unexplained variability was included using either proportional or combined proportional/additive error models.

The final model was selected based on likelihood ratio test with $p<0.05$, equivalent to a decrease of the objective function value of 3.84; visual predictive check (VPC) plots to compare the model fit to drug concentrations in plasma; precision of the parameter estimates denoted by the \%relative standard errors; and the basic goodness of fit plots that include individual/population predictions versus observations and conditional weighted residuals versus population prediction/time.

Human plasma PK models were either available from the literature or developed in a similar fashion as described for rats.

\section{LeiCNS-PK3.0 evaluation}

LeiCNS-PK3.0 model performance was evaluated using visual prediction check plots (VPCs), where the median and $95 \%$ prediction interval of 200 model simulations were plotted against and compared to in vivo-measured unbound drug concentrations. The model simulations accounted for interindividual variability and residual variabilities of the plasma PK model, as described above. The relevant $\eta$ of interindividual variability and $\varepsilon$ of residual unexplained variabilities were randomly sampled from a normal distribution with a mean of 0 and a variance of $\omega^{2}$ and $\sigma^{2}$, respectively, and transformed as required.

Next, prediction errors were calculated using the individual measured drug concentrations and their corresponding time-matched simulations median. Average fold error (AFE) was calculated to evaluate the model's bias, 
while absolute average fold error (AAFE) was calculated to compare the typical PK profile simulated by the model to the typical PK profile of the measured PK data. A typical profile is the profile predicted assuming no interindividual variability, i.e. when etas are set to zero. AFE and AAFE were calculated using relative accuracy calculated for each drug. AFE and AAFE values approaching 100\% denote accurate model predictions.

Relative Accuracy of a given drug $\left(\mathrm{RA}_{\text {drug }}\right)$ at a given compartment was calculated as follows:

$$
\begin{aligned}
& R A_{d r u g}=\frac{1}{M} \sum_{i=1}^{N} \sum_{j=1}^{m} \log _{10}\left(\frac{M e d P_{i, j}}{O b s_{i, j}}\right) \\
& M=\sum_{i=1}^{N} m
\end{aligned}
$$

where $\mathrm{Obs}_{\mathrm{i}, \mathrm{j}}$ is $\mathrm{jth}$ observation of the ith individual; $\mathrm{MedP}_{\mathrm{i}, \mathrm{j}}$ is the median value of the 200 simulations corresponding to $\mathrm{Obs}_{\mathrm{i}, \mathrm{j}}$; $\mathrm{M}$ is the total number of observations of all individuals; $m$ is the number of observations of the ith individual; and $\mathrm{N}$ is the total number of individuals.

$\%$ AFE of a given compartment was calculated as:

$A F E=\frac{1}{D} \sum_{d=1}^{D} R A_{d r u g}$

$\% A F E=100 \times 10^{A F E}$

where D is the number of drugs used for evaluation.

$\%$ AAFE of a given compartment was calculated as:

$A A F E=\frac{1}{D} \sum_{d=1}^{D}\left|R A_{d r u g}\right|$

$\% A A F E=100 \times 10^{A A F E}$

In addition, the mean absolute relative accuracy (MARA) was calculated to evaluate the variability of individual drug concentrations around the median of LeiCNS-PK3.0 simulations within a given compartment. MARA was based on absolute relative accuracy of a given drug $\left(\mathrm{ARA}_{\mathrm{drug}}\right)$ at a given compartment, which was calculated as:

$A R A_{\text {drug }}=\frac{1}{M} \sum_{i=1}^{N} \sum_{j=1}^{m}\left|\log _{10}\left(\frac{M e d P_{i, j}}{O b s_{i, j}}\right)\right|$

$M A R A=\frac{1}{D} \sum_{d=1}^{D} A R A_{d r u g}$

$\%$ MARA $=100 \times 10^{\text {MARA }}$

where $\mathrm{Obs}_{\mathrm{i}, \mathrm{j}}$ is $\mathrm{jth}$ observation of the ith individual; $\mathrm{MedP}_{\mathrm{i}, \mathrm{j}}$ is the median value of the 200 simulations corresponding to $\mathrm{Obs}_{\mathrm{i}, \mathrm{j}}$; $\mathrm{M}$ is the total number of observations of all individuals; $m$ is the number of observations of the ith individual; $\mathrm{N}$ is the total number of individuals; and $\mathrm{D}$ is the number of drugs used for evaluation.

Symmetric mean absolute prediction errors (SMAPE) were calculated to benchmark LeiCNS-PK3.0 with LeiCNS-PK1.0. A SMAPE value closer to $0 \%$ implies a more accurate model.

$\operatorname{SMAPE}(\%)=\frac{100}{M} \sum_{i=1}^{N} \sum_{j=1}^{m}\left|\frac{2 *\left(O b s_{i, j}-\operatorname{MedP} P_{i, j}\right)}{O b s_{i, j}+\operatorname{MedP} P_{i, j}}\right|$

where $\mathrm{Obs}_{\mathrm{i}, \mathrm{j}}$ is $\mathrm{jth}$ observation of the ith individual; $\mathrm{MedP}_{\mathrm{i}, \mathrm{j}}$ is the median value of the 200 simulations corresponding to $\mathrm{Obs}_{\mathrm{i}, \mathrm{j}} ; \mathrm{M}$ is the total number of observations of all individuals; $m$ is the number of observations of the ith individual; and $\mathrm{N}$ is the total number of individuals.

\section{The effect of altered CSF dynamics on brain ECF PK}

The effect of altered CSF volume and flow on the drug exposure in the brain ECF and CSF was studied using human LeiCNS-PK3.0. Simulations were performed for six drugs with different physicochemical properties. Test drugs included methotrexate, acetaminophen, phenytoin, atenolol, raclopride, and risperidone. A fixed 1-compartment plasma PK model of human was applied across all drugs in order to isolate the impact of CSF parameters from other variables. Rat $\mathrm{Kp}_{\mathrm{uu}}$ values and the associated $\mathrm{AF}$ were adapted for humans. The resulting drug concentration ratio of brain ECF-to-SAS was compared between the physiological, two- and five-fold CSF volume and flow. SAS in this setting represents lumbar CSF PK profile, while brain ECF represents the brain PK profile, assuming no active transport takes place at the level of the brain cells. Brain $\mathrm{ECF}$ is an intermediate compartment between brain microvasculature and brain cells and therefore unaltered drug exposure in brain ECF will imply unaltered drug exposure in brain cells. Two- and five-folds changes were selected to reflect the changes of CSF volume and CSF flow in CNS diseases as reported in Table 1. For example, the volume of the ventricles increase by $4.57 \%$ /year during healthy aging [31], which in the course of 20 years will result in the expansion of the ventricles to about $250 \%$. The CSF flow, measured at the aqueduct of patients with idiopathic normal pressure hydrocephalus patients, increases to $370 \%$ of its physiological value [32].

\section{Sensitivity analysis}

A sensitivity analysis was performed using the human version of LeiCNS-PK3.0 to identify the main parameters that define the PK profiles at the brain ECF, brain ICF and SAS. The sensitivity analysis was carried out using four 
drugs with distinct physicochemical properties: acetaminophen, morphine, methotrexate, and raclopride. The CNS parameters were varied individually by $1.1,1.5$, and 2 folds, and resulting PK descriptors, $\mathrm{C}_{\max }, \mathrm{T}_{\max }$, and AUC, in the selected compartments were compared to those of the physiological situation, using the sensitivity index calculated as:

Sensitivity index $=\log _{2} \frac{Y_{d}}{Y_{o}}$

where $Y_{d}$ and $Y_{o}$ are the pharmacokinetic descriptors $\left(\mathrm{C}_{\max }, \mathrm{T}_{\max }\right.$, and $\left.\mathrm{AUC}\right)$ of the altered and physiological values, respectively.

\section{Data analysis and software}

Plasma PK model parameters were estimated using NONMEM version 7.4.3 (ICON, Dublin, Ireland) [33]. General data analysis and visualization and LeiCNS-PK3.0 simulations were performed using $\mathrm{R}$ version 3.6.1 [34], where simulations were performed using RxODE package version 0.9.1-0 [35], using the LSODA (Livermore Solver for Ordinary Differential Equations) Fortran package. Algebraic equations were solved using Maxima Computer Algebra System version 19.01.2x (available from http:// maxima.sourceforge.net). Literature data were extracted with WebPlotDigitizer version 4.2 (https://apps.automeris. io/wpd/).

\section{Results}

\section{Plasma PK models}

The empirical plasma model parameters of the rat and human are displayed in Table 4. Rat plasma PK model parameters were estimated with good precision and the models accurately described the observed plasma drug concentrations. The plasma PK model of methotrexate, however, slightly overpredicted the data. Human plasma models of acetaminophen and morphine were available from the literature [36], while plasma PK model parameters of oxycodone and indomethacin were developed.

\section{Model evaluation}

The CNS model of LeiCNS-PK3.0 was developed using bottom-up modeling relying on physiological information only. Evaluation of the model predictions was performed using published PK data from different brain regions, and thus model evaluation is independent from model development.

\section{Rat LeiCNS-PK3.0 evaluation}

Figure 2 and Supplementary Fig. 2a-b depict the VPC plots of rat LeiCNS-PK3.0 simulations against the measured drug concentrations of 10 drugs (Supplementary table 1). LeiCNS-PK3.0 adequately predicted the observed data in the brain ECF, lateral ventricles (LV), and cisterna magna (CM), with some exceptions. Methotrexate brain ECF and quinidine $20 \mathrm{mg} \mathrm{LV}$ concentrations were slightly underpredicted. Phenytoin brain ECF and CM and quinidine $\mathrm{CM}$ concentrations were underpredicted towards the end of the simulation. Remoxipride 4, 8,16 mg predictions captured the peak of the observations but overpredicted the remaining observations. LeiCNS-PK3.0 additionally predicted brain homogenate $(\mathrm{BH})$ concentrations, but less adequately. The model overpredicted quinidine and remoxipride $0.7 \mathrm{mg}$ and underpredicted phenytoin $40 \mathrm{mg}$ observations and raclopride peak concentration.

In addition, LeiCNS-PK3.0 performance was evaluated by calculating the relative accuracy error and its derivatives: \%AFE and \%AAFE that assess the model's bias and typical PK profile predictability, respectively. Supplementary Fig. 2C displays a box plot of relative accuracy errors. \%AFE (95\% confidence interval) of brain ECF, LV, CM and $\mathrm{BH}$ were $90 \%(67-120), 77 \% \quad(41-146), 80 \%$ (56-116), and 64\% (6-643), respectively. These values deviate by a maximum of $35 \%$ from the optimum value of $100 \%$ and are indeed within two-fold error. \%AAFE $(95 \%$ confidence interval) were $140 \%$ (118-167), 139\% (85229), and $149 \%$ (120-185) for brain ECF, LV and CM, respectively, which deviate by $<50 \%$ and are within twofold error. BH predictions were less accurate, with a $\%$ AAFE of $322 \%$ (99-1045).

SMAPEs, besides, were calculated for comparison with LeiCNS-PK1.0. SMAPE of LeiCNS-PK3.0 (vs LeiCNSPK1.0) were $65 \%$ (vs $72 \%$ ), $71 \%$ (vs $71 \%$ ), $70 \%$ (vs 69\%), and $105 \%$ (vs $91 \%$ ) for brain ECF, LV and $\mathrm{CM}$ and $\mathrm{BH}$, respectively.

\section{Human LeiCNS-PK3.0 evaluation}

Figure 3 displays the VPC plots of the human LeiCNSPK3.0 simulations against the measured concentrationtime profiles of four drugs (Supplementary table 1). The plots show that LeiCNS-PK3.0 adequately predicted the brain ECF and SAS concentrations. Acetaminophen and indomethacin SAS concentration were underpredicted to some extent. \%AFE (Supplementary Fig. 3) of brain ECF and SAS were $92 \%$ and $56 \%$, respectively. \%AAFE of brain ECF and SAS were $109 \%$ and $179 \%$, respectively. All error values were within the two-fold error limit. 


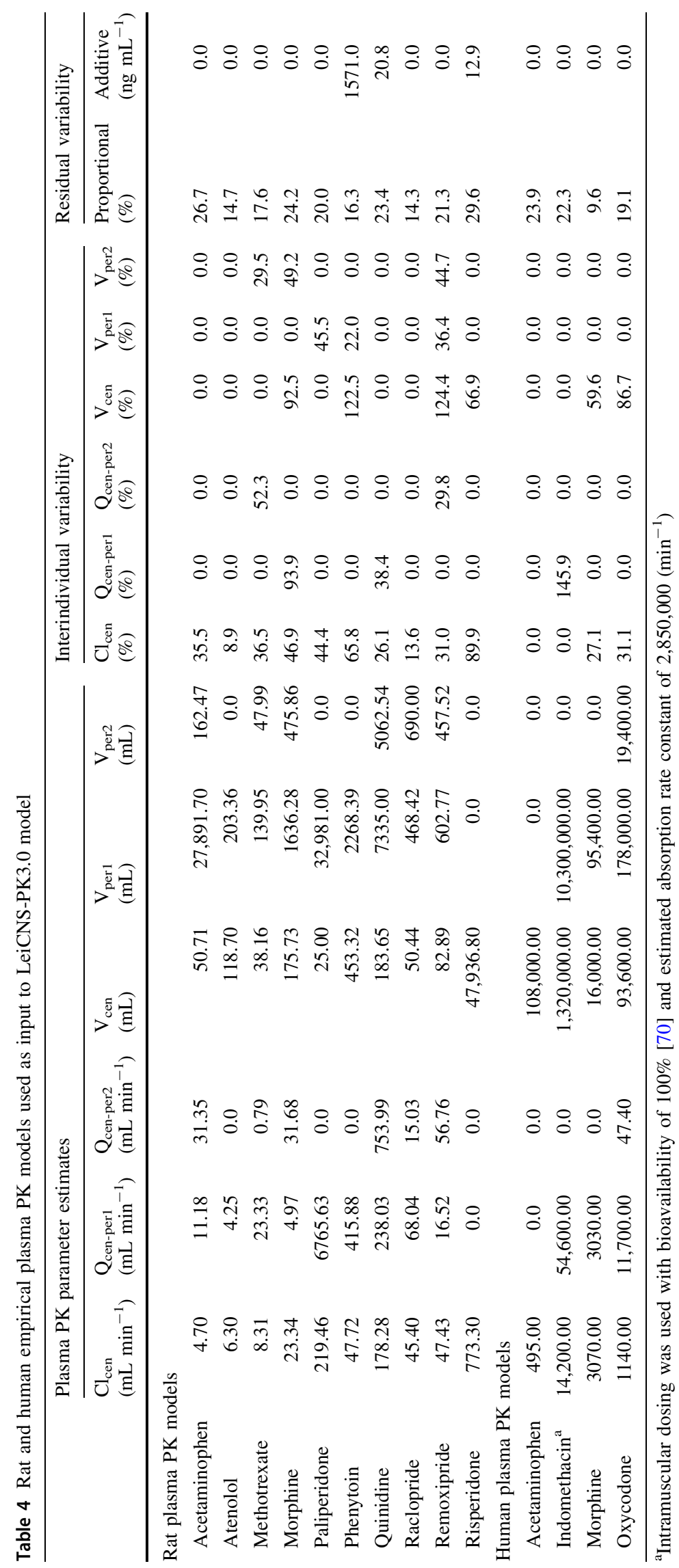




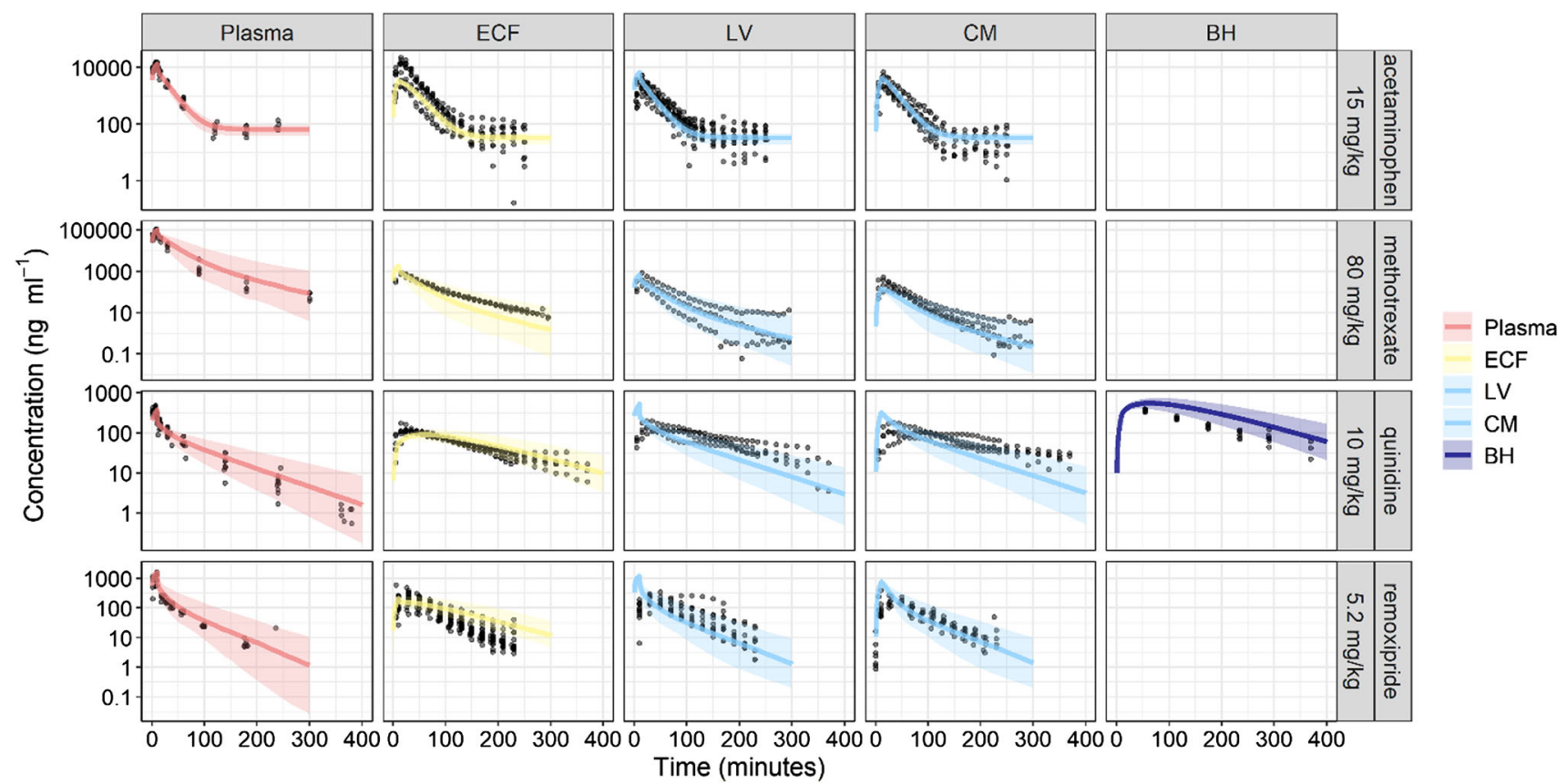

Fig. 2 Model evaluation of the rat LeiCNS-PK3.0 model. Visual predictive checks plots compared in vivo measured drug concentration (black dots) in multiple CNS locations to the median (solid line) and $95 \%$ prediction intervals (colored band) of 200 model simulations. $E C F$ brain extracellular fluid, $L V$ lateral ventricles, $C M$ cisterna magna, $B H$ brain homogenate (Color figure online)
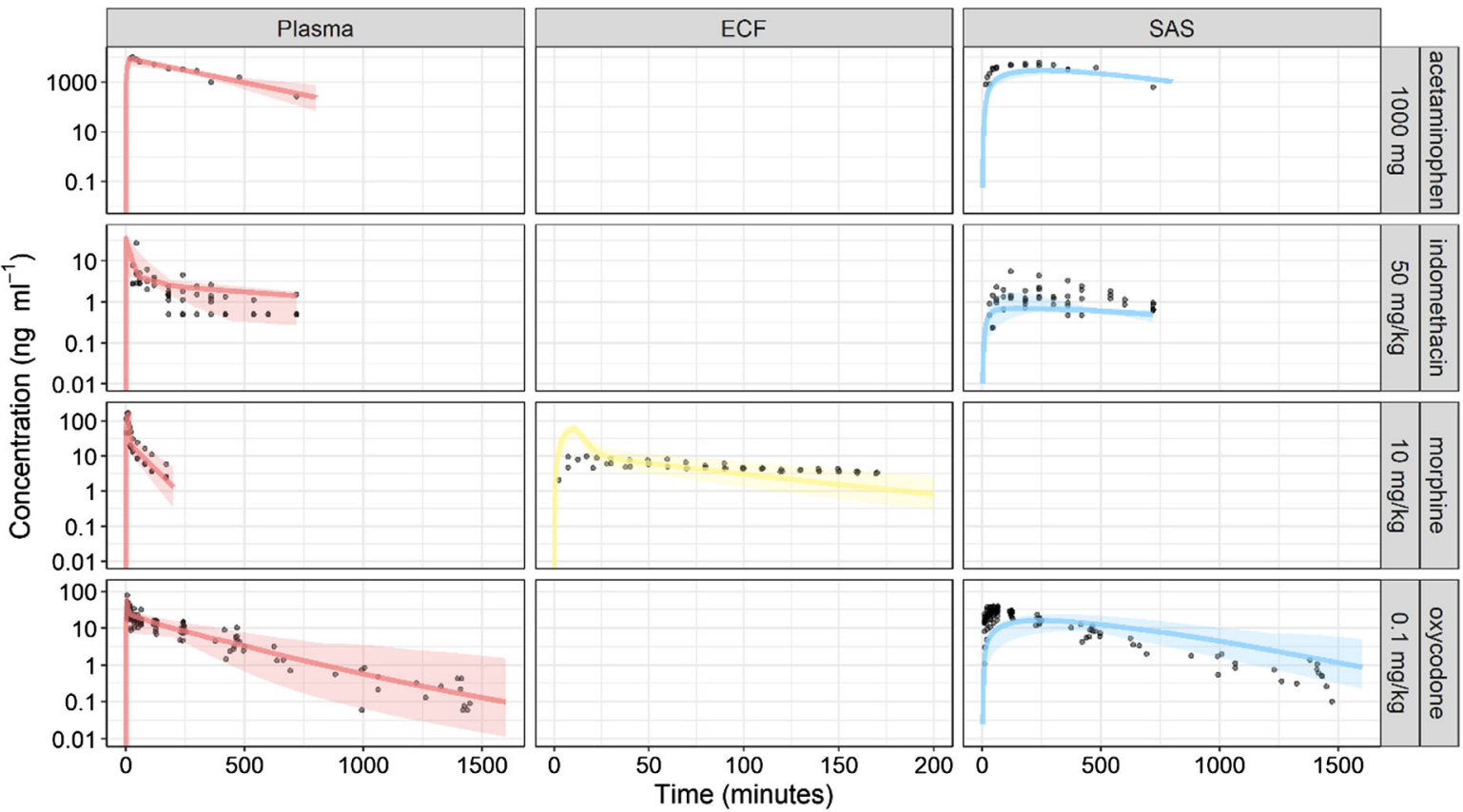

Plasma

ECF

SAS

Fig. 3 Model evaluation of the human LeiCNS-PK3.0 model. Visual predictive checks plots compared in vivo measured drug concentration (black dots) in multiple CNS locations to the median (solid line)

\section{Effect of altered CSF dynamics on brain ECF and CSF pharmacokinetics}

PK profiles of brain ECF and SAS compartments at different CSF flow and volumes are shown in Fig. 4a, b for and $95 \%$ prediction intervals (colored band band) of 200 model simulations. ECF brain extracellular fluid, $S A S$ subarachnoid space (Color figure online)

acetaminophen and Supplementary Fig. 4 a-e and 5 a-e for methotrexate, phenytoin, atenolol, raclopride, and risperidone. Changes in CSF volume and flow altered SAS but not brain ECF PK profile and hence changed the brain ECF-SAS ratio. Within the SAS, decrease in CSF volume 

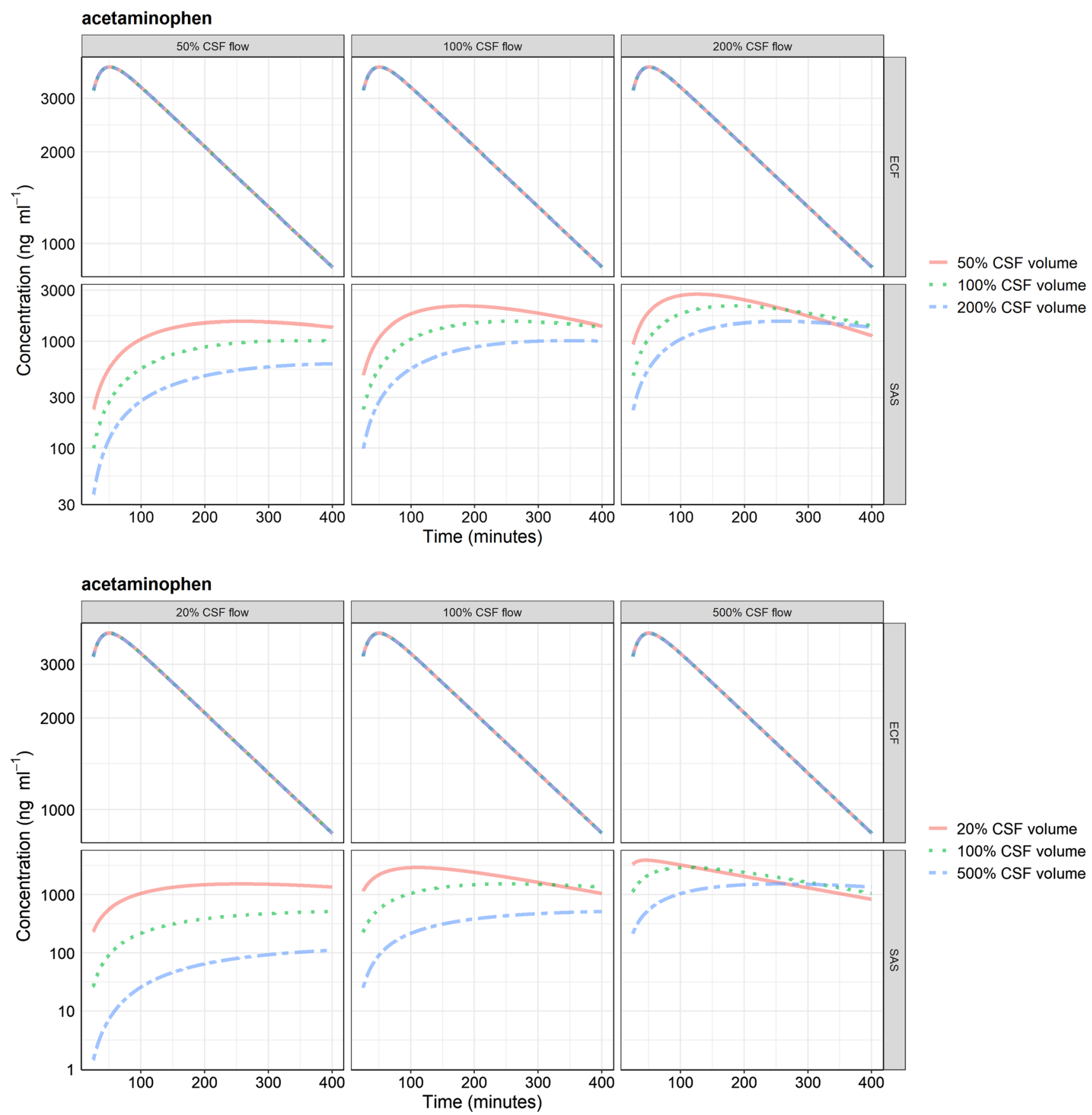

- $20 \%$ CSF volume

" - $100 \%$ CSF volume

- $=500 \%$ CSF volume

Fig. 4 Pharmacokinetic profiles of acetaminophen at brain extracellular (ECF) fluid and subarachnoid space (SAS) at physiological and a two- and $\mathbf{b}$ five-fold altered cerebrospinal fluid (CSF) volume and

or increase in CSF flow results in an earlier $\mathrm{T}_{\max }$, higher $\mathrm{C}_{\max }$, and a faster clearance. The observed changes of $\mathrm{T}_{\max }$ and $\mathrm{C}_{\max }$ at the SAS compartment was the same for all drugs regardless their physicochemical properties. flow. Changing CSF dynamics affects SAS pharmacokinetics and not brain ECF pharmacokinetics. ECF brain extracellular fluid, SAS subarachnoid space

\section{Sensitivity analysis}

LeiCNS-PK3.0 sensitivity analysis was performed to identify the CNS model parameters that influence the PK profiles at the brain ECF, brain ICF, and SAS. The identified parameters were drug- and CNS compartment-dependent. Brain ECF and ICF PK profiles were sensitive to active transport at $\mathrm{BBB}$ as reflected by brain-to-plasma 
unbound drug partitioning ( $\left.\mathrm{Kp}_{\mathrm{uu}, \mathrm{ECF}}\right)$, volume and surface area of brain cells, width of BBB and tight junction pore, and $\mathrm{pH}$ of brain ECF and ICF. The SAS PK profile was sensitive to active transport at BCSFB given by the CSFto-plasma unbound drug partitioning $\left(\mathrm{Kp}_{\mathrm{uu}, \mathrm{CM}}\right)$, CSF flow, and SAS volume. LeiCNS-PK3.0 sensitivity analysis results are shown in Supplementary Fig. 6.

\section{Discussion}

LeiCNS-PK3.0 simulations showed that altered CSF dynamics resulted in a shift in the drug concentration ratio of brain ECF-to-SAS CSF, where SAS CSF PK profiles but not brain ECF PK profiles were affected. This observation is independent of the drug's physicochemical properties, as it is assumed in the model that transport into and out of the SAS CSF is mediated by CSF flow and does not involve barrier transport. This implies a context-specific surrogacy of lumbar CSF-to-brain ECF PK profiles and thus this relationship is not suitable for interpopulation or interspecies translation. LeiCNS-PK3.0 simulations, thus, reproach the classical assumption of the prediction of lumbar CSF drug concentration to brain ECF drug concentrations [2], which is in line with previous findings [3].

\section{LeiCNS-PK3.0 performance}

LeiCNS-PK3.0 is an improved and a more mechanistic version of LeiCNS-PK1.0 [6, 7], where the physiological processes of non-specific binding and $\mathrm{pH}$ effect on drug ionization and passive transport across BBB and BCSFB have been addressed. LeiCNS-PK3.0 predictions are based exclusively on plasma PK, CNS physiological parameters, drug physicochemical properties, and in vitro measurements. LeiCNS-PK3.0 predicts brain non-specific binding using a drug property, i.e. lipophilicity, which is either measured at the early stages of drug development or predicted with QSAR approaches. This makes lipophilicity more efficient to use compared to the formerly-used brain unbound drug fraction $\left(\mathrm{f}_{\mathrm{u}, \mathrm{b}}\right)$, which requires brain tissue.

LeiCNS-PK3.0 predictions are predominantly unbiased as indicated by the below 35\% \%AFE. The model, however, slightly underpredicts drug concentrations of human SAS, but within the two-fold error margin. Drug concentration-time profiles of rat brain ECF, LV, and CM and of human brain ECF and SAS were adequately predicted. \%AAFE errors, which indicate the model prediction of typical PK profiles, were within the two-fold error limit, with human brain ECF predictions deviating less than $10 \%$.

LeiCNS-PK3.0 predicted BH PK profiles less adequately which could be the result of the unaccounted for physiological processes such as brain metabolism, active transport at the brain cells, specific binding of drugs to target receptor, etc. BH predictions of raclopride, a known dopamine D2 receptor substrate [37], displayed the largest error among other drugs. \%AAFE of $\mathrm{BH}$ without including raclopride was $223 \%$ compared to $322 \%$ with raclopride. Future inclusion of receptor binding and other physiological process is anticipated to improve LeiCNS-PK3.0 predictions.

LeiCNS-PK3.0 predictions of human brain intracellular fluid (ICF) PK profiles are depicted in supplementary Figs. 8. Both brain ECF and ICF represent the sites of drug action, which makes their PK profiles of top interest to drug developers. Brain ICF PK profiles cannot be validated with in vivo PK profiles, as such data are not attainable. Imaging techniques do not distinguish intracellular and extracellular drug. The brain slice method could be used to investigate the concentration and time dependency of the equilibrium between the brain ECF, represented by the buffer, and brain ICF [38]. This in vitro method is, however, limited by the loss of the whole brain context as a number of physiological processes such as bulk flows are missed, in addition to the limited duration of tissue viability.

\section{Sensitivity analysis: implications to LeiCNS-PK3.0 assumptions}

A number of LeiCNS-PK3.0 parameters were calculated based on certain assumptions about CNS physiology, some of which were found by the sensitivity analysis to largely affect CNS PK. The affected assumptions were: surface of the brain cells membrane $\left(\mathrm{SA}_{\mathrm{BCM}}\right), \mathrm{CSF}$ flow, and active transport.

$\mathrm{SA}_{\mathrm{BCM}}$ was calculated using brain cells volume and number, assuming that all brain cells are spheres of equal radii. CSF flow was assumed constant in ventricles and the subarachnoid space, which does not reflect the physiology. Active transport was accounted for by calculating AF using $\mathrm{Kp}_{\mathrm{uu}}$ whose value is dependent on dosing and measurement techniques. Improving the mechanistic description of these parameters should be a priority of future investigations and will increase the confidence in LeiCNS-PK3.0 predictions.

\section{New non-specific binding model}

Brain non-specific binding in LeiCNS-PK3.0 is presented as a time-dependent process; a diffusion clearance describes the drug partitioning between brain ECF/ICF and phospholipids of the brain cell membrane. This is based on two assumptions. First, phospholipids of the brain cell membrane play a determinant role in non-specific binding within brain compared to the negligible role of brain proteins e.g. albumin [23, 39], neutral lipids [20], and other components of brain cells [22]. The second assumption 
relates to $\mathrm{P}_{\mathrm{oct}-\mathrm{w}}$ representation of biological lipophilicity. Octanol-water system represents a simplified model of drug partitioning between aqueous and lipid phases, compared to the phospholipid bilayer of the brain cell membrane. $\mathrm{P}_{\mathrm{oct}-\mathrm{w}}$, for example, neglects the partitioning of charged molecules to phospholipids. A number of studies have demonstrated the correlation of $\mathrm{P}_{\mathrm{oct}-\mathrm{w}}$ and brain nonspecific binding. $\mathrm{P}_{\text {oct-w }}$ was shown to explain about $52 \%$ (reported as $\mathrm{R}^{2}$ ) of the variability in experimentally-measured volume of distribution of unbound drug $\left(\mathrm{V}_{\mathrm{u}, \text { brain }}\right)$ in brain [14] and about $44-74 \%$ (reported as $\mathrm{R}^{2}$ ) of the variability in experimentally-measured fraction of unbound drug in brain $\left(f_{u, b}\right)$ [11-13]. This evidence indicates that $\mathrm{P}_{\text {oct-w }}$ provides an adequate predictor of brain non-specific binding.

\section{pH effect on drug ionization and its effect on drug transendothelial transport}

Drug molecules in the CNS ionize depending on the compartment-specific $\mathrm{pH}$ and the drug-specific acid and base ionization constants. In LeiCNS-PK3.0, it is assumed that charged molecules can cross the barriers by paracellular diffusion only, ignoring the transcellular transport of charged species and paracellular route preference to cationic drugs [8]. Charged drug transcellular and paracellular transport rate is, however, negligible compared to neutral species transport rate and is not expected to critically influence LeiCNS-PK3.0 predictions.

\section{In vivo studies addressing the impact of CSF dynamics on brain ECF versus CSF PK profiles}

A number of studies have supported the surrogacy of the CSF PK profiles to those of brain ECF, based on studies performed in rats, for both actively and passively transported drugs [2, 40, 41]. These studies are based, however, on CSF samples collected at the cisterna magna. Cranial CSF, including CSF at the cisterna magna, is in a relatively faster equilibrium with brain ECF, as compared to the distal lumbar CSF. In contrast to what is generally assumed, it has been shown in both in silico [42] and preclinical and clinical studies [43] that lumbar CSF does not reflect the PK profiles of brain ECF or even cisternal and ventricular CSF. In addition, our LeiCNS-PK3.0 sensitivity analysis suggests that brain ECF and ICF pharmacokinetic profiles are insensitive to CSF-related parameters. In a similar modeling study, the sensitivity analysis of a permeability-limited CNS PBPK model demonstrated that multiple factors while affecting the PK profiles of lumbar CSF, did not affect those of brain or even cranial CSF [42].
Preclinical and clinical studies that address the impact of altered CSF volume and/or flow on brain CSF PK profiles are rare, due to the associated technical and ethical restrictions. In addition, changing one CNS parameter in isolation is more of a hypothetical situation rather than can truly be realized in in vivo studies. Notwithstanding, a number of studies have addressed the impact of acetazolamide-induced reduction of CSF flow on brain ECF and CSF PK profiles. Acetazolamide is a carbonic anhydrase inhibitor drug, which reduces CSF production and flow by about $50 \%$. Methotrexate exposure in the ventricular CSF of three patients was altered following acetazolamide administration, where the terminal elimination half-life increased [44] in agreement with the altered simulated profiles in Fig. 4 and supplementary Fig. 4 of this manuscript. The PK profile of alovudine measured in rat brain ECF with microdialysis was not altered in response to acetazolamide co-administration [45]. The PK profiles of 5-fluorouracil at rat brain ECF and cisterna magna CSF were altered to different extents following acetazolamide administration, implying the context dependency of drug concentration ratio of brain ECF to CSF [46]. It can be concluded as supported by LeiCNS-PK3.0 simulations and the in vivo preclinical and clinical studies that the lumbar CSF to brain ECF drug concentration ratio is context-dependent and this ratio might be altered in response to a change in CSF dynamics.

\section{Absence of CNS IIV and its implications}

LeiCNS-PK3.0 accounts for interindividual variability (IIV) of the plasma pharmacokinetic parameters, but not that of the CNS physiology parameters. The impact of the IIV of CNS parameters on PK profiles is more prominent when drug transport is dependent on a certain parameter. For example, acetaminophen's, a slightly lipophilic and paracellularly-transported molecule, brain ECF PK profile is sensitive to the tight junction pore diameter (Supplementary Fig. 6). Thus, IIV of the tight junction pore diameter might account for the larger observed variability of brain ECF PK profile compared to that of plasma (Fig. 2, top panel). Acetaminophen PK profile while assuming nominal variabilities of $30 \%$ and $50 \%$ (as coefficient of variation, \%CV) on physiological CNS parameters showed slightly wider 2.5 th and 97.5 th percentiles, which therefore better described the observed variability of the PK data (Supplementary Fig. 7).

The variability of the individual observed CNS concentrations relative to typical predicted profile was within three-fold error as indicated by \%MARA. For humans, \%MARA errors were $182 \%, 238 \%$ for brain ECF and SAS, respectively, while for rats these were $207 \%, 229 \%$, and $216 \%$ for brain ECF, LV, and CM, respectively. 
Identification of variability of CNS model parameters and associated covariates is crucial for predicting the individual PK profiles, which remains challenging due to the limited data, e.g. on CNS physiology and measured drug concentrations, required for estimating this level of variability.

\section{Patho-pharmacokinetics require a systems approach}

CNS drug exposure in healthy and diseased conditions is a function of both physiological and drug properties. In a healthy CNS, a number of mechanisms contribute to the rate and extent of the actual drug transport across the BBB, resulting in a brain ECF PK profile that may substantially differ from that of plasma. A change in any of the parameters that govern the PK at brain ECF and ICF, as identified by the sensitivity analysis, would potentially result in altered CNS drug exposure. This is particularly crucial in CNS diseases, in which the complex and multifactorial disease-specific pathophysiology would result in a distinct CNS PK profiles compared to those of a healthy CNS. In epilepsy, for instance, the increased expression of active efflux transporters at BBB is associated with a lower drug exposure in brain and hence resistance to therapy [47]. Furthermore, patients with traumatic brain injury showed higher morphine concentrations of the injured brain tissue ECF than those of the uninjured tissue, which is potentially due to decreased tight junction and active transporters expression at the BBB $[7,48]$. Mechanistic, systems-based approaches such as PBPK modeling account for drug and CNS physiological properties in addition to the multidimensional disease pathology and are thus better suited for adequate PK predictions in healthy and diseased CNS. The shortage of knowledge on (patho-) physiological parameters and mechanisms remains a major challenge to translating CNS PBPK models between healthy and diseased populations.

\section{LeiCNS-PK3.0 applications}

LeiCNS-PK3.0 applications include predicting PK profiles of small drugs in a healthy CNS and in patients with CNS diseases, e.g. Alzheimer's, and exploring mechanistically the impact of CNS disease pathophysiology on CNS PK i.e. patho-pharmacokinetics. These applications are supported by mechanistic detailing of different physiological processes that for example distinguishes paracellular and transcellular transports, but also accounts for brain cells and lysosomes, a feature that was not supported in similar published CNS models [42, 49, 50]. LeiCNS-PK3.0 is thus useful at early stages of drug development to support (pre-) clinical study design and decision-making, e.g. dose selection and sampling time points.

\section{Conclusion}

In conclusion, we improved our published LeiCNS-PK1.0 by accounting for brain non-specific binding and readdressing $\mathrm{pH}$ effect on drug ionization and passive transport. LeiCNS-PK3.0 simulations demonstrated that altered CSF dynamics changes brain ECF-to-SAS drug concentration ratio and implied a context-dependent PK surrogacy of lumbar SAS to brain ECF.

Author contributions MAAS, JE-S, and ECMDL contributed to the project design and model improvement. MAAS and CFL performed data collection and model simulations. MAAS, JE-S, and ECM de Lange contributed to manuscript writing.

Funding No funding was received to assist with the preparation of this manuscript.

Data availability Data generated during and analyzed during the current study are not publicly available due privacy but are available from the corresponding author on reasonable request.

\section{Declarations}

Conflict of interest The authors have no conflicts of interest to declare that are relevant to the content of this article.

Supplementary Information The online version contains supplementary material available at https://doi.org/10.1007/s10928021-09768-7.

Open Access This article is licensed under a Creative Commons Attribution 4.0 International License, which permits use, sharing, adaptation, distribution and reproduction in any medium or format, as long as you give appropriate credit to the original author(s) and the source, provide a link to the Creative Commons licence, and indicate if changes were made. The images or other third party material in this article are included in the article's Creative Commons licence, unless indicated otherwise in a credit line to the material. If material is not included in the article's Creative Commons licence and your intended use is not permitted by statutory regulation or exceeds the permitted use, you will need to obtain permission directly from the copyright holder. To view a copy of this licence, visit http://creativecommons. org/licenses/by/4.0/.

\section{References}

1. Cook D, Brown D, Alexander R et al (2014) Lessons learned from the fate of AstraZeneca's drug pipeline: a five-dimensional framework. Nat Rev Drug Discov 13:419-431. https://doi.org/10. 1038/nrd4309

2. Liu X, Smith BJ, Chen C et al (2006) Evaluation of cerebrospinal fluid concentration and plasma free concentration as a surrogate measurement for brain free concentration. Drug Metab Dispos 34:1443-1447. https://doi.org/10.1124/dmd.105.008201

3. De Lange ECM (2013) Utility of CSF in translational neuroscience. J Pharmacokinet Pharmacodyn 40:315-326. https://doi. org/10.1007/s10928-013-9301-9 
4. Srinivas N, Maffuid K, Kashuba ADM (2018) Clinical pharmacokinetics and pharmacodynamics of drugs in the central nervous system. Clin Pharmacokinet 57:1059-1074. https://doi.org/10. 1007/s40262-018-0632-y

5. Vendel E, Rottschäfer V, De Lange ECM (2019) The need for mathematical modelling of spatial drug distribution within the brain. Fluids Barriers CNS 16:12. https://doi.org/10.1186/s12987019-0133-X

6. Yamamoto Y, Välitalo P, Huntjens D et al (2017) Predicting drug concentration-time profiles in multiple relevant CNS compartments using a comprehensive physiologically-based pharmacokinetic model. CPT Pharmacometrics Syst Pharmacol 6:765-777. https://doi.org/10.1002/psp4.12250

7. Yamamoto Y, Välitalo PA, Wong YC et al (2018) Prediction of human CNS pharmacokinetics using a physiologically-based pharmacokinetic modeling approach. Eur J Pharm Sci 112:168-179. https://doi.org/10.1016/j.ejps.2017.11.011

8. Anderson JM (2001) Molecular structure of tight junctions and their role in epithelial transport. News Physiol Sci 16:126-130. https://doi.org/10.1152/physiologyonline.2001.16.3.126

9. Fridén M, Bergström F, Wan H et al (2011) Measurement of unbound drug exposure in brain: Modeling of $\mathrm{pH}$ partitioning explains diverging results between the brain slice and brain homogenate methods. Drug Metab Dispos 39:353-362. https:// doi.org/10.1124/dmd.110.035998

10. Liu X, Vilenski O, Kwan J et al (2009) Unbound brain concentration determines receptor occupancy: a correlation of drug concentration and brain serotonin and dopamine reuptake transporter occupancy for eighteen compounds in rats. Drug Metab Dispos 37:1548-1556. https://doi.org/10.1124/dmd.109.026674

11. Summerfield SG, Read K, Begley DJ et al (2007) Central nervous system drug disposition: the relationship between in situ brain permeability and brain free fraction. J Pharmacol Exp Ther 322:205-213. https://doi.org/10.1124/jpet.107.121525

12. Esaki T, Ohashi R, Watanabe R et al (2019) Computational model to predict the fraction of unbound drug in the brain. J Chem Inf Model 59:3251-3261. https://doi.org/10.1021/acs. jcim.9b00180

13. Wan H, Åhman M, Holmén AG (2009) Relationship between brain tissue partitioning and microemulsion retention factors of CNS drugs. J Med Chem 52:1693-1700. https://doi.org/10.1021/ jm801441s

14. Spreafico M, Jacobson MP (2013) In silico prediction of brain exposure: drug free fraction, unbound brain to plasma concentration ratio and equilibrium half-life. Curr Top Med Chem 13:813-820. https://doi.org/10.2174/1568026611313070004

15. De Lange ECM, Hammarlund-Udenaes M (2015) Translational aspects of blood-brain barrier transport and central nervous system effects of drugs: from discovery to patients. Clin Pharmacol Ther 97:380-394. https://doi.org/10.1002/cpt.76

16. Fridén M, Winiwarter S, Jerndal G et al (2009) Structure-brain exposure relationships in rat and human using a novel data set of unbound drug concentrations in brain interstitial and cerebrospinal fluids. J Med Chem 52:6233-6243. https://doi.org/10. 1021/jm901036q

17. Wishart DS, Feunang YD, Guo AC et al (2017) DrugBank 5.0: a major update to the DrugBank database for 2018. Nucleic Acids Res 46:D1074-D1082. https://doi.org/10.1093/nar/gkx1037

18. Mannhold R, Poda GI, Ostermann C, Tetko IV (2009) Calculation of molecular lipophilicity: state-of-the-art and comparison of LogP methods on more than 96,000 compounds. J Pharm Sci 98:861-893. https://doi.org/10.1002/jps

19. Manchester J, Walkup G, Rivin O, You Z (2010) Evaluation of pka estimation methods on 211 drug-like compounds. J Chem Inf Model 50:565-571. https://doi.org/10.1021/ci100019p
20. Treyer A, Mateus A, Wiśniewski JR et al (2018) Intracellular drug bioavailability: effect of neutral lipids and phospholipids. Mol Pharm 15:2224-2233. https://doi.org/10.1021/acs.mol pharmaceut.8b00064

21. Mateus A, Matsson P, Artursson P (2013) Rapid measurement of intracellular unbound drug concentrations. Mol Pharm 10:2467-2478. https://doi.org/10.1021/mp4000822

22. Mateus A, Treyer A, Wegler C et al (2017) Intracellular drug bioavailability: a new predictor of system dependent drug disposition. Sci Rep. https://doi.org/10.1038/srep43047

23. Poulin P, Theil FP (2000) A priori prediction of tissue: plasma partition coefficients of drugs to facilitate the use of physiologically-based pharmacokinetic models in drug discovery. J Pharm Sci 89:16-35. https://doi.org/10.1002/(SICI)15206017(200001)89:1\%3c16::AID-JPS3\%3e3.0.CO;2-E

24. Poulin P, Theil F-P (2002) Prediction of pharmacokinetics prior to in vivo studies. 1. Mechanism-based prediction of volume of distribution. J Pharm Sci 91:129-156. https://doi.org/10.1002/jps. 10128

25. Hammarlund-Udenaes M, Fridén M, Syvänen S, Gupta A (2008) On the rate and extent of drug delivery to the brain. Pharm Res 25:1737-1750. https://doi.org/10.1007/s11095-007-9502-2

26. Ball K, Bouzom F, Scherrmann J-M et al (2013) physiologically based pharmacokinetic modelling of drug penetration across the blood-brain barrier-towards a mechanistic IVIVE-based approach. AAPS J 15:913-932. https://doi.org/10.1208/s12248013-9496-0

27. Chen X, Slättengren T, De Lange ECM et al (2017) Revisiting atenolol as a low passive permeability marker. Fluids Barriers CNS 14:1-14. https://doi.org/10.1186/s12987-017-0078-x

28. Norris MD, De Graaf D, Haber M et al (1996) Involvement of MDR1 P-glycoprotein in multifactorial resistance to methotrexate. Int J Cancer 65:613-619. https://doi.org/10.1002/(SICI)10970215(19960301)65:5\%3c613::AID-IJC10\%3e3.0.CO;2-8

29. Volk EL, Schneider E (2003) Wild-type breast cancer resistance protein (BCRP/ABCG2) is a methotrexate polyglutamate transporter. Cancer Res 63:5538-5543

30. Bai J, Lai L, Yeo HC et al (2004) Multidrug resistance protein 4 (MRP4/ABCC4) mediates efflux of bimane-glutathione. Int $\mathrm{J}$ Biochem Cell Biol 36:247-257. https://doi.org/10.1016/S13572725(03)00236-X

31. Fjell AM, McEvoy L, Holland D et al (2013) Brain changes in older adults at very low risk for Alzheimer's disease. J Neurosci 33:8237-8242. https://doi.org/10.1523/JNEUROSCI.5506-12. 2013

32. Lindstrøm EK, Ringstad G, Mardal K-AA, Eide PK (2018) Cerebrospinal fluid volumetric net flow rate and direction in idiopathic normal pressure hydrocephalus. NeuroImage Clin 20:731-741. https://doi.org/10.1016/j.nicl.2018.09.006

33. Bauer RJ (2019) NONMEM users guide: introduction to NONMEM 7.4.3. ICON Plc, Gaithersburg

34. Team RC (2019) R: a language and environment for statistical computing. R Foundation for Statistical Computing, Vienna

35. Fidler M, Hallow M, Wilkins J, Wang W (2019) RxODE: facilities for simulating from ode-based models. $\mathrm{R}$ package version 1.0.9

36. Yamamoto Y, Välitalo PA, van den Berg DJ et al (2017) A generic multi-compartmental CNS distribution model structure for 9 drugs allows prediction of human brain target site concentrations. Pharm Res 34:333-351. https://doi.org/10.1007/ s11095-016-2065-3

37. Wong YC, Ilkova T, van Wijk RC et al (2018) Development of a population pharmacokinetic model to predict brain distribution and dopamine D2 receptor occupancy of raclopride in nonanesthetized rat. Eur J Pharm Sci 111:514-525. https://doi.org/10. 1016/j.ejps.2017.10.031 
38. Fridén M, Gupta A, Antonsson M et al (2007) In vitro methods for estimating unbound drug concentrations in the brain interstitial and intracellular fluids. Drug Metab Dispos 35:1711-1719. https://doi.org/10.1124/dmd.107.015222

39. Longhi R, Corbioli S, Fontana $S$ et al (2010) Brain tissue binding of drugs: evaluation and validation of solid supported porcine brain membrane vesicles (TRANSIL) as a novel high-throughput method. Drug Metab Dispos 39:312-321. https://doi.org/10.1124/ dmd.110.036095

40. Liu X, Van Natta K, Yeo H et al (2009) Unbound drug concentration in brain homogenate and cerebral spinal fluid at steady state as a surrogate for unbound concentration in brain interstitial fluid. Drug Metab Dispos 37:787-793. https://doi.org/10.1124/ dmd.108.024125

41. Xiao G, Black C, Hetu G et al (2012) Cerebrospinal fluid can be used as a surrogate to assess brain exposures of breast cancer resistance protein and P-glycoprotein substrates. Drug Metab Dispos 40:779-787. https://doi.org/10.1124/dmd.111.043703

42. Gaohua L, Neuhoff S, Johnson TN, Rostami-hodjegan A (2016) Development of a permeability-limited model of the human brain and cerebrospinal fluid (CSF) to integrate known physiological and biological knowledge: estimating time varying CSF drug concentrations and their variability using in vitro data. Drug Metab Pharmacokinet 31:224-233. https://doi.org/10.1016/j. dmpk.2016.03.005

43. Lin JH (2008) CSF as a surrogate for assessing CNS exposure: an industrial perspective. Curr Drug Metab 9:46-59. https://doi.org/ 10.2174/138920008783331077

44. Bode U, Magrath IT, Bleyer WA et al (1980) Active transport of methotrexate from cerebrospinal fluid in humans. Cancer Res 40:2184-2187

45. Ståhle L, Borg N (2000) Transport of alovudine (3'-fluorothymidine) into the brain and the cerebrospinal fluid of the rat, studied by microdialysis. Life Sci 66:1805-1816. https://doi.org/ 10.1016/S0024-3205(00)00504-X

46. Shingaki T, Hidalgo IJ, Furubayashi T et al (2009) The transnasal delivery of 5-fluorouracil to the rat brain is enhanced by acetazolamide (the inhibitor of the secretion of cerebrospinal fluid). Int J Pharm 377:85-91. https://doi.org/10.1016/j.ijpharm.2009.05. 009

47. Löscher W, Potschka H (2005) Blood-brain barrier active efflux transporters: ATP-binding cassette gene family. NeuroRx 2:86-98. https://doi.org/10.1602/neurorx.2.1.86

48. Ederoth P, Tunblad K, Bouw R et al (2004) Blood-brain barrier transport of morphine in patients with severe brain trauma. Br J Clin Pharmacol 57:427-435. https://doi.org/10.1046/j.1365-2125. 2003.02032.x

49. Trapa PE, Belova E, Liras JL et al (2016) Insights from an integrated physiologically based pharmacokinetic model for brain penetration. J Pharm Sci 105:965-971. https://doi.org/10.1016/j. xphs.2015.12.005

50. Kielbasa W, Stratford RE (2012) Exploratory translational modeling approach in drug development to predict human brain pharmacokinetics and pharmacologically relevant clinical doses. Drug Metab Dispos 40:877-883. https://doi.org/10.1124/dmd. 111.043554

51. Ito M, Hatazawa J, Yamaura H, Matsuzawa T (2014) Age-related brain atrophy and mental deterioration - a study with computed tomography. Br J Radiol 54:384-390. https://doi.org/10.1259/ 0007-1285-54-641-384

52. Tanna NK, Kohn MI, Horwich DN et al (1991) Analysis of brain and cerebrospinal fluid volumes with MR imaging: impact on PET data correction for atrophy. Part II. Aging and Alzheimer Dementia. Radiology 178:123-130. https://doi.org/10.1148/radi ology.178.1.1984290
53. Hodel J, Lebret A, Petit E et al (2013) Imaging of the entire cerebrospinal fluid volume with a multistation 3D SPACE MR sequence: feasibility study in patients with hydrocephalus. Eur Radiol 23:1450-1458. https://doi.org/10.1007/s00330-012-27327

54. Konstantinou N, Pettemeridou E, Seimenis I et al (2016) Assessing the relationship between neurocognitive performance and brain volume in chronic moderate-severe traumatic brain injury. Front Neurol 7:29. https://doi.org/10.3389/fneur.2016. 00029

55. May C, Kaye JA, Atack JR et al (1990) Cerebrospinal fluid production is reduced in healthy aging. Neurology 40:500-503

56. Silverberg GD, Heit G, Huhn S et al (2001) The cerebrospinal fluid production rate is reduced in dementia of the Alzheimer's type. Neurology 57:1763-1766

57. Silverberg GD, Huhn S, Jaffe RA et al (2002) Downregulation of cerebrospinal fluid production in patients with chronic hydrocephalus. J Neurosurg 97:1271-1275. https://doi.org/10.3171/jns. 2002.97.6.1271

58. Oner Z, Kahraman AS, Kose E et al (2017) Quantitative evaluation of normal aqueductal cerebrospinal fluid flow using phasecontrast cine MRI according to age and sex. Anat Rec 300:549-555. https://doi.org/10.1002/ar.23514

59. El Sankari S, Gondry-Jouet C, Fichten A et al (2011) Cerebrospinal fluid and blood flow in mild cognitive impairment and Alzheimer's disease: a differential diagnosis from idiopathic normal pressure hydrocephalus. Fluids Barriers CNS 8:12. https://doi.org/10.1186/2045-8118-8-12

60. Nagra G, Johnston MG (2007) Impact of ageing on lymphatic cerebrospinal fluid absorption in the rat. Neuropathol Appl Neurobiol 33:684-691. https://doi.org/10.1111/j.1365-2990. 2007.00857.x

61. de Leon MJ, Li Y, Okamura N et al (2017) Cerebrospinal fluid clearance in alzheimer disease measured with dynamic PET. J Nucl Med 58:1471-1476. https://doi.org/10.2967/jnumed.116. 187211

62. Hakim R, Black PM (1998) Correlation between lumbo-ventricular perfusion and MRI-CSF flow studies in idiopathic normal pressure hydrocephalus. Surg Neurol 49:14-20. https://doi.org/ 10.1016/S0090-3019(97)00032-3

63. Reid G, Wielinga P, Zelcer $\mathrm{N}$ et al (2003) The human multidrug resistance protein MRP4 functions as a prostaglandin efflux transporter and is inhibited by nonsteroidal anti inflammatory drugs. Proc Natl Acad Sci U S A 100:9244-9249. https://doi.org/ 10.1073/pnas. 1033060100

64. Iliás A, Urbán Z, Seidl TL et al (2002) Loss of ATP-dependent transport activity in pseudoxanthoma elasticum-associated mutants of human ABCC6 (MRP6). J Biol Chem 277:16860-16867. https://doi.org/10.1074/jbc.M110918200

65. Jung KY, Takeda M, Kim DK et al (2001) Characterization of ochratoxin A transport by human organic anion transporters. Life Sci 69:2123-2135. https://doi.org/10.1016/S00243205(01)01296-6

66. Cha SH, Sekine T, Fukushima JI et al (2001) Identification and characterization of human organic anion transporter 3 expressing predominantly in the kidney. Mol Pharmacol 59:1277-1286. https://doi.org/10.1124/mol.59.5.1277

67. Uchida Y, Ohtsuki S, Katsukura Y et al (2011) Quantitative targeted absolute proteomics of human blood-brain barrier transporters and receptors. J Neurochem 117:333-345. https:// doi.org/10.1111/j.1471-4159.2011.07208.x

68. Al Feteisi H, Al-Majdoub ZM, Achour B et al (2018) Identification and quantification of blood-brain barrier transporters in isolated rat brain microvessels. J Neurochem 146:670-685. https://doi.org/10.1111/jnc.14446 
69. Al-Majdoub ZM, Al Feteisi H, Achour B et al (2019) Proteomic quantification of human blood-brain barrier SLC and ABC transporters in healthy individuals and dementia patients. Mol Pharm 16:1220-1233. https://doi.org/10.1021/acs.molpharma ceut.8b01189

70. Jensen KM, Grenabo L (1985) Bioavailability of indomethacin after intramuscular injection and rectal administration of solution and suppositories. Acta Pharmacol Toxicol (Copenh) 57:322-327. https://doi.org/10.1111/j.1600-0773.1985.tb00052.x

Publisher's Note Springer Nature remains neutral with regard to jurisdictional claims in published maps and institutional affiliations. 